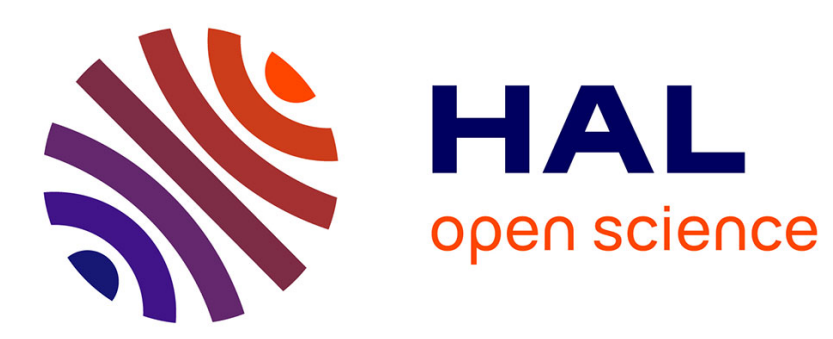

\title{
De nouveaux modèles de virilité: musiques actuelles et cultures urbaines
}

\author{
Yves Raibaud
}

\section{To cite this version:}

Yves Raibaud. De nouveaux modèles de virilité: musiques actuelles et cultures urbaines. WelzerLang, Daniel; Zaouche-Gaudron, Chantal. Masculinités: état des lieux, Erès, pp.169-181, 2011, 10.3917/eres.welze.2011.01.0149 . halshs-00596743

\section{HAL Id: halshs-00596743 https://shs.hal.science/halshs-00596743}

Submitted on 30 May 2011

HAL is a multi-disciplinary open access archive for the deposit and dissemination of scientific research documents, whether they are published or not. The documents may come from teaching and research institutions in France or abroad, or from public or private research centers.
L'archive ouverte pluridisciplinaire HAL, est destinée au dépôt et à la diffusion de documents scientifiques de niveau recherche, publiés ou non, émanant des établissements d'enseignement et de recherche français ou étrangers, des laboratoires publics ou privés. 


\section{Yves Raibaud}

\section{De nouveaux modèles de virilité: musiques actuelles et cultures urbaines}

Maître de conférences, HDR, Université de Bordeaux, ADES-CNRS,y.raibaud@ades.cnrs.fr 10, Chemin du Vieux Château, 33420 Dardenac. Tél : 0556239516 ; 0672736292.

Cet article a pour source un travail de recherche réalisé en France sur les musiques actuelles (rock, rap, techno, reggae...) et plus généralement sur les cultures urbaines (danse hip-hop, graff, sports urbains tels que skate, rollers, bmx), à partir du constat que ces pratiques sont très majoritairement masculines ${ }^{1}$.

La diffusion mondiale des musiques populaires anglosaxonnes dans les décennies 1960 et 1970 est à l'origine de pratiques musicales de masse, globalement regroupées sous le terme générique de rock au début des années $1980^{2}$. Le fait que ces nouvelles pratiques culturelles soient l'expression des jeunes, en particulier ceux qui viennent des classes populaires et des quartiers fragiles, est alors un fait tenu pour acquis. Cela favorise dans les années 1990 leur intégration dans le dispositif interministériel de la politique de la ville, entre prévention, politique de la jeunesse et culture. De nouveaux équipements voient le jour (centres et écoles de musiques amplifiées, salles de musiques actuelles) ${ }^{3}$; des réseaux départementaux ou régionaux s'organisent et diffusent un modèle d'action sociale et culturelle s'appuyant sur ces cultures émergentes, bientôt appelées «cultures urbaines ». Les équipements musicaux consacrés à ces cultures sont en effet contemporains de nouveaux modèles d'architecture urbaine destinés aux loisirs des jeunes tels que skates parcs et cités stades (ou citystades).

L'étude de ces lieux peut contribuer à une meilleure connaissance sociologique du « masculin» pour au moins trois raisons que nous développerons dans cet article. La

\footnotetext{
1 MSHA/Ministère de la Culture 2003, ADES-Université de Bordeaux 2007, 2009.

2 En 1982, un recensement effectué à la demande du ministre Jack Lang dénombre 30000 groupes de rock sur l'ensemble du territoire français. La rencontre entre les pouvoirs publics et les leaders du rock est rendue possible par l'appellation de «musique amplifiée »: ce n'est pas une catégorie culturelle qui est mise en avant, mais un ensemble de pratiques sociales et culturelles caractérisées par l'amplification du son, qui nécessite des lieux de répétitions et de concerts adaptés pour ne pas troubler l'ordre public.

${ }^{3}$ Telle le Rocher de Palmer à Cenon, salle nationale des musiques actuelles inaugurée en septembre 2010, qui s est rendu célèbre récemment par la programmation du groupe de rap sexiste et homophobe Sexion d `Assaut, alors que la tournée du groupe avait été annulée dans 17 autres villes de France (voir fin d article).
} 
première est leur homogénéité de genre $(85 \%$ d'hommes pour les pratiques de musiques actuelles, jusqu'à 100\% dans les skates parcs et cités stades) et le fait que cette homogénéité est masquée par la neutralité du discours qui définit ces lieux et leur public, permettant l'occultation des pratiques sexuées qu'ils recèlent. La deuxième est la projection sur l'espace de cette inégalité de genre, montrant comment ces pratiques participent à la création d'idéologies territoriales fonctionnelles associant les cultures urbaines à un univers légendaire de la ville et de ses périphéries: le territoire mythique de la banlieue apparaît dans ces conditions comme un lieu fonctionnel pour l'apparition de nouvelles cultures masculines et leur diffusion dans la société toute entière. La troisième raison est que les cultures urbaines se réfèrent de façon fréquente aux cultures ethniques et/ou noires (jazz, rock, reggae, rap pour la musique, hip-hop pour la danse, street basket et sports de rue pour le sport) tout en étant pratiquées et consommées par toutes les catégories de population. Le constat que la "'race' colore le genre » (Dorlin, 2006) dans ces nouvelles cultures masculines permet de poser l'hypothèse que les musiques actuelles et les cultures urbaines fonctionnent comme des « opérateurs hiérarchiques » (Latour in Welzer-Lang [2004], Ayral [2009]) sur le double registre du genre et de l`ethnicité, organisant ainsi les systèmes de sens nécessaires aux nouvelles formes de régulation sociale et spatiale contemporaines.

\section{Des pratiques majoritairement masculines}

La première recherche menée sur les musiques amplifiées entre 2000 et 2003 avait montré que ces pratiques étaient majoritairement masculines. En 2009, si les centres et écoles de musiques amplifiées ${ }^{4}$ semblent enregistrer une progression de la fréquentation féminine hors concert (environ 20\%), la présence de celles-ci est concentrée sur les cours de musique, et particulièrement les cours de chant. Elles ne sont plus que 10\% dans les activités répétitions et environ 5\% dans les prestations sur scène, groupes amateurs et professionnels confondus. Les centres de musiques amplifiées sont dirigés par des hommes, 85\% du personnel permanent est masculin, idem pour les conseils d'administration des associations qui organisent ces centres. Les représentants des collectivités et de l'Etat qui participent à leur financement sont des hommes, en général volontaires pour ce travail en raison d'affinités culturelles avec le monde du rock. Les documents de présentation des centres sont rédigés au masculin neutre (les musiciens, les techniciens, les animateurs, le coordinateur) sauf pour 
l'activité chant (les chanteurs-euses).

L'activité la plus valorisée est le prêt de locaux gratuits ou faiblement payants à des groupes de jeunes musiciens auto-organisés. Des cours de musique, un studio d enregistrement et la possibilité de se produire sur scène en première partie d artistes reconnus complète cette offre culturelle subventionnée, présentée comme une proposition alternative à l'offre jugée élitiste et conservatrice des écoles de musique. En France, d autres pratiques musicales amateurs sont mixtes et peu ou pas subventionnées : chorales, orchestres d'harmonie, mais aussi bandas et batucadas qui ont les faveurs d'un public jeune (Raibaud, 2005 b). Mon propos consiste donc à m'interroger sur la double particularité des musiques amplifiées-actuelles: des pratiques masculines d une part, considérées comme d'utilité sociale d autre part.

On retrouve ces caractéristiques dans d autres équipements construits en grand nombre dans les deux dernières décennies: les skates parcs et les cités stades. Des statistiques réalisées sur les pratiques sportives montraient que la participation des femmes plafonnait depuis plusieurs années à $30 \%$ de 1 o offre de loisirs publique, toutes pratiques confondues ${ }^{5}$, malgré les importants horaires d éducation sportive dispensés dans l'enseignement public. Le constat que ces moyennes sont plus faibles encore pour les femmes en fonction de leur classe sociale et de leur lieu d'habitation (quartiers classés zone urbaine sensible par exemple) n'a pas empêché que soit déclenchée une vaste opération de construction de terrains de sports d'accès libres fréquentés exclusivement par les garçons, notamment dans les quartiers relevant de la politique de la ville. L étude en cours sur les équipements de la Communauté Urbaine de Bordeaux enregistre près de $100 \%$ de jeunes hommes sur les cités stades ${ }^{6}$ et $90 \%$ sur les skates parcs. Sur les skates parcs, une minorité de jeunes filles en skate s impose à égalité avec les garçons (avec un haut niveau de performance), une autre minorité (roller) fréquente en groupe de filles les équipements à des heures spécifiques (skate parc des quais, Bordeaux). Voici le résumé d une observation ethnographique des deux skates parcs de la ville de G., dans la banlieue de Bordeaux :

\footnotetext{
sociale.

5 Chiffres obtenus en cumulant des pratiques mixtes/non mixtes (sports collectifs ou athlétisme) et les pratiques non mixtes spécifiques telles que la gymnastique rythmique et sportive ou la danse (Femmes et sport, rapport2005, site du MJSVA).

6 Conçus pour la polyvalence des pratiques sportives, les cités stades sont utilisés principalement comme des terrains de foot, de façon marginale comme des terrains de basket.
} 
Les skate-parcs de G.

G. est une commune de la périphérie de Bordeaux marquée par des taux supérieurs à la moyenne en termes d’habitat social, chômage des jeunes, familles monoparentales, population étrangère. Ce ňest cependant pas une commune très marquée par la violence des jeunes et lünsécurité. On peut penser que cela est dû au fait que les municipalités successives ont mis en place depuis des années des politiques d̆animation et de prévention. Lüiée qu il convient de canaliser la violence des jeunes vers des activités positives est le credo de la mairie et des professionnel.le.s.

La création de skates parcs et de cités stades d accès libre répond à ce souci et est plébiscitée par les jeunes. Ľobservation des skates parcs de G. montre qu ils sont fréquentés presque exclusivement par des garçons. Les femmes contournent largement ce lieu, certaines jeunes filles y viennent avec leur copain attitré ou entre copines proches de la bande des garçons pour les regarder. Les filles qui pratiquent le skate (5\%) sont bien acceptées car elles sont en général performantes et qu elles ont adopté les tenues et les comportements des garçons. Draguer les filles qui sont assises sur les murets est mal vu car elles ne viennent en général que si elles sont déjà avec un garçon (celles qui viennent seules sont censées " chercher l'aventure »).

Les garçons ne portent pas de protection et se défient, au risque de la chute et de la blessure. Les garçons maladroits ou peu sportifs qui ne peuvent pas montrer des signes extérieurs de virilité évitent le lieu, plus encore que les filles. Dans un des skates parcs de la ville, une piste junior a été construite pour les petits. Les mamans y emmènent leurs petits garçons le mercredi et le dimanche après-midi, montrant bien que ces nouvelles pratiques ont été intégrées dans la formation des garçons, au même titre que les sports collectifs ou les arts martiaux. Enfin dans un quartier de G., le skate parc est devenu un lieu de rencontre nocturne entre dealers et jeunes consommateurs de drogues. (Raibaud, 2007b). 
D autres activités, recensées sous le nom de «cultures urbaines », présentent les mêmes caractéristiques, avec cependant des variations : le graff est très masculin (Felonneau, 2001), la danse l’est moins grâce à la diffusion des pratiques hip-hop dans les cours de danse. Les festivals de cultures urbaines (Festival des Cultures Urbaines, Paris-La Villette, Vibrations urbaines, Pessac etc.) sont des festivals masculins ${ }^{7}$, même si les programmateurs

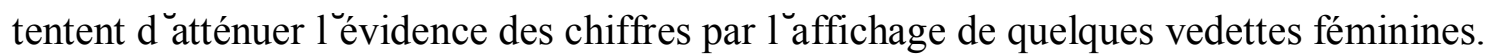

\section{Négation des chiffres $=$ occultation des pratiques}

A la question posée «pourquoi y a-t-il si peu de filles dans ces équipements? », les responsables des structures de musiques actuelles (des hommes) répondent d'abord par la négation : les chiffres sont faux, il y a beaucoup de filles. Ensuite, dès lors qu'il est admis que les chiffres sont justes, ils montrent le rôle actif de la minorité : les filles sont moins nombreuses, mais actives et il y en a « de plus en plus ». Lorsqu'on peut démontrer que le « de plus en plus » est relatif, les responsables des équipements temporisent : l'important, ce sont les jeunes qui sont écartés des loisirs et de la culture (les jeunes des quartiers, issus de l'immigration par exemple). De plus, les filles ont des équipements spécifiques pour elles (les salles de danse, de gym). L'idée qu'il convient de canaliser la violence des jeunes dans des activités d'expression est première, et cela concerne principalement les jeunes garçons, mais que peut-on y faire ? N'est-ce pas eux qui posent problème?

Mêmes réponses du côté des élu.e.s et chefs de service (des hommes), en charge de la politique de la ville, concernant les skates parcs et les cités stades. Les pratiques sportives urbaines anarchiques (roller, skate) sont rapprochées d autres actes de petite délinquance tels que rodéo en scooter dans les jardins publics, tags, concerts de tambours nocturnesǔ Ces jeunes ne viennent pas dans les équipements sportifs et culturels traditionnelsǔ Le skate parc et le cité stade sont des lieux de socialisation qui peuvent les amener par la suite à fréquenter d ăatres équipements... Où sont les filles ? Il y en a « de plus en plus » sur les skates parcs et les cités stadesǔ mais la plupart préfèrent, c ěst bien normal, des activités plus féminines comme la danse, ou bien elles choisissent le travail d école et rester à la maisonǔ

Ce déni des chiffres sert décran à la question que poserait le constat de pratiques uniquement masculines et de ce qu èlles produisent. Nous avons montré dans un précédent

\footnotetext{
7 Hommes sur scène ou sur les terrains, présence minoritaire de femmes dans le public.
} 
article comment un lieu de répétition des musiques amplifiées pouvait être modélisé comme une « maison-des-hommes» (Godelier, in Welzer-Lang, 2004), créatrice de formes renouvelées de cultures masculines fondées en particulier sur lexpression du sexisme et de 1 homophobie (Raibaud, 2007 a). Les skates parcs de G. peuvent être analysés comme des lieux masculins tout aussi hétéronormatifs, mais représentant une autre variation culturelle de la maison-des-hommes : au contraire des lieux de répétitions fermés où le sexe est présent dans les noms de groupe, les textes, les blagues, léchange de revues et de films pornographiques, les skates parcs sont neutres ou faiblement érotisés pour les garçons. Plutôt anxiogènes pour la majorité des femmes qui les évitent, ils peuvent être également agréables pour certaines car s y donne à voir la «masculinité comme noblesse » (Bourdieu, 1998). L ăttention des garçons se porte en effet moins sur les filles que sur la compétition qui les oppose entre eux.

La négation (par les responsables, les élu.e.s) du caractère exclusivement masculin de ces lieux occulte les pratiques masculines qui y ont cours. Ceci interroge alors non pas ces pratiques elles-mêmes, mais les politiques publiques qui les encouragent. Le souci du maintien de lordre public tend donc à socialiser les jeunes garçons de façon préventive à 1 intérieur d espaces masculins hétéronormatifs. Lorsqu ils sont situés dans des quartiers $\mathrm{d}$ habitat social périphérique, les équipements des cultures urbaines (en particulier skates parcs et cités stades) s inscrivent souvent dans la continuité d espaces dans lesquels les hommes sont dominants. La virilité étant un attribut des hommes dans leurs relations au monde, la question de leur présence dans les espaces publics pose alors problème (Kebabza, 2004).

Les préconisations apportées par les professionnels de l־aménagement urbain (sociologues ou urbanistes) procèdent souvent d une approche hétéronormative construite sur le principe d égalité des sexes, qui consiste à répartir les espaces genrés selon les rôles sociaux attribués classiquement aux femmes et aux hommes : d un côté des maisons, parcs et jardins publics avec jeux pour les enfants protégés de la culture « lascar», des rodéos, des attitudes agressives; de 1 a autre côté des salles de concerts, skates parks, cités stades pour éviter lenvahissement de la ville par les skateurs, rappeurs, breakers, sliders et adeptes de performances spectaculaires le plus souvent associées à la compétition virile. Canaliser la violence des garçons dans des espaces d expression : une grande partie de la gestion urbaine, dans son appréhension des loisirs des jeunes, repose sur ce présupposé. 


\section{Des idéologies territoriales fonctionnelles}

Une autre façon d ăborder les musiques amplifiées et les cultures urbaines consiste à les appréhender sous l⿳ăangle de leurs productions culturelles. J `ai relaté dans plusieurs articles et ouvrages les analyses artistiques et textuelles qui montraient comment les producteurs des cultures urbaines (organisateurs dévénements, animateurs d ăteliers, responsables de salles) participaient à la construction d un imaginaire artistique et culturel de la banlieue. Le quartier relégué, doté d une morphologie fonctionnelle (au-delà d une bretelle d autoroute, à droite du fleuve, en bas d une colline, doté d un habitat en hauteur), est mis en scène par la valorisation des caractéristiques socioculturelles d une partie de sa population (pauvre, immigrée, à la peau noire ou foncée), ce qui permet aisément de le catégoriser en référence aux ghettos urbains des grandes mégapoles mondiales. Le classement d un grand nombre de ces quartiers en zone urbaine sensible a permis dans les années 1990 en France la mise en place dune action culturelle spécifique de grande ampleur fondée sur les musiques actuelles (dans leur version « musiques du monde ») et les cultures urbaines (Mendjeli et Raibaud, 2008).

On pourrait d une façon synthétique dégager trois types de récits performatifs qui participent à la création de ces imaginaires culturels, dont un des effets attendus est de « réenchanter » la banlieue. Le premier récit, šappuyant sur 1 homogénéité de la banlieue comme territoire dominé, affirme que la créativité culturelle se renouvelle dans les luttes sociales. La banlieue serait un lieu d innovation. Le deuxième récit suppose que la banlieue est populaire, interculturelle et métissée (donc authentique et joyeuse ?), ce qui l`opposerait à 1 hégémonie de la ville centre et de ses lieux culturels (tristes ?), symboles de la domination culturelle de la bourgeoisie et de 1 aristocratie. Le troisième récit attribue un pouvoir magique à la culture pour réguler les tensions et canaliser la violence sociale.

La diffusion des cultures urbaines mondiales par les médias stigmatise fortement 1 espace des banlieues dans un contexte de métropolisation et de hiérarchisation des territoires urbains $^{8}$. L action culturelle menée dans les quartiers par les entrepreneurs des cultures urbaines vient apporter un surplus d identification aux quartiers fragiles en les mettant en contact avec des artistes représentant lexpression culturelle des ghettos des grandes mégapoles. Les quartiers, porteurs des signes et attributs de cet imaginaire hip-hop, sont reflétés comme tels par les médias qui couvrent ces manifestations culturelles : immeubles et 
grillages, population masculine à la peau noire ou foncée, garçons vêtus de vêtements amples, casquettes de base-ball et baskets, musiciens rap... (plaquette de présentation du festival «Quartiers Musiques », in Raibaud, 2005 a). La culture des quartiers est ainsi consommée par tous, sans distinction. Récente, il lui est pour 1 instant nécessaire d être authentifiée par le parcours des artistes qui la produisent (éventuellement par la différence visible que représente la couleur de leur peau) et illustrée par le décor des cités et des grands ensembles dont la connotation « violences urbaines » est quotidiennement rappelée par les médias.

Certains pensent que les difficultés économiques et le chômage, aggravant la relégation des garçons des quartiers, accentueraient leur position difficile face aux images viriarcales de la réussite (Coutras, 2003): les cultures urbaines seraient alors une voie possible de réussite leur permettant de restaurer une image positive d eux mêmes. Même s íl convient de nuancer cette interprétation «classique » en prenant en compte la diversité des situations individuelles des garçons (notamment les moins «virils»), les quartiers fragiles apparaissent comme un lieu favorable pour que s épanouissent les références à de nouvelles cultures masculines agissant comme compensation. Le quartier devient ainsi le lieu symbolique de la re-production des attitudes viriles.

\section{Des références ethniques}

Les municipalités socialistes de la rive droite de Bordeaux que nous avons interrogées sont tentées de faire la promotion de leur territoire à travers ces actions. L idée que les cultures urbaines sont susceptibles de donner une image de jeunesse et de dynamisme à leurs territoires n ěst cependant pas évidente et č est la vocation sociale de ces manifestations qui apparaît en premier, contribuant un peu plus à létiquetage de ces parties de la ville. Il est peu probable que l`identification des quartiers fragiles de la rive droite de Bordeaux aux favelas de Rio via la programmation du groupe brésilien Moleque de Rua ou aux ghettos de

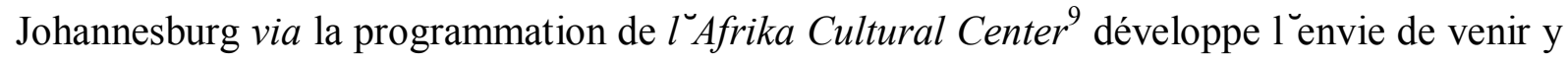
résider, alors que les communes périphériques de la rive gauche (plus favorisées) mettent des moyens importants pour soutenir localement une consommation culturelle caractérisant les classes moyennes et supérieures. L observation des discours et des pratiques des animateurstrices socioculturel.le.s en banlieue permet de poser 1 hypothèse inverse de la permanence des 
processus de reproduction sociale et culturelle issus de lexpérience coloniale, et des injonctions paradoxales du discours intégrationniste. Le politologue Rachid Mendjeli (Mendjeli, 2003, 2008) explique ainsi comment les musiques actuelles et les cultures urbaines agissent comme ersatz de « la culture d origine des populations immigrées » en participant à la construction du discours politique sur le métissage culturel. L offre d identification symbolique et politique qu elles proposent passe en effet par la mise en scène des références à 1 histoire, aux normes et aux valeurs des «cultures monde » comme porte-drapeaux des revendications des cultures populaires dominées. Les cultures urbaines sont transposées dans les quartiers $d$ habitats sociaux comme le reflet des situations de domination sociale locale des populations immigrées. Cette proximité sociale et culturelle entre les cultures urbaines et les cultures des populations dites immigrées contribuerait, selon les animateurs-trices culturel.le.s, à la production de normes d identification et de mobilisation culturelle «des jeunes de banlieues ».

Ainsi, dans un pays où la dimension ethnique des phénomènes culturels est officiellement niée, laaction des pouvoirs publics participe au maintien de cette ethnicité au profit de populations qui ne sont pas majoritairement étrangères, mais considérées comme telles car issues des anciennes colonies. On peut certes signaler la capacité d artistes sortis des lieux décrits à s emparer des modèles proposés et à les détourner à leur profit. Mais on peut aussi y voir leefficacité performative du discours qui amène ces détournements à référer en permanence au labelling des quartiers. L hypothèse $\mathrm{d}$ un double bind (entre encouragement à la création culturelle et assignation à une identité culturelle ethnique de quartier) nous fait imaginer que cette situation contribue à produire un système sociospatial spécifique, intégré et revendiqué par les plus jeunes auxquels ces discours s ădressent et au moyen desquels ils seront contrôlés ${ }^{10}$.

\section{Les cultures urbaines comme opérateur hiérarchique de genre et de :race־}

Plutôt que d analyser les intersections et/ou les causalités entre masculinité et ethnicité à travers ces pratiques culturelles et sportives urbaines ${ }^{11}$, il nous est apparu important de poser 1 hypothèse que les cultures urbaines fonctionnent comme un actant ou opérateur et agissent comme un "quasi personnage ", "non seulement un objet dont on parle mais un sujet

d authenticité et de créativité musicale.

10 Situation décrite par Didier Lapeyronnie, dans Ghetto Urbain, 2008.

11 On pourrait évoquer les généalogies de ces rapports comme «matrices » de la nation française (Dorlin, 2006) dans 
agissant dans le discours $»^{12}$. Le fait de réunir dans la catégorie cultures urbaines les musiques actuelles/amplifiées, les musiques du monde, la culture hip-hop et les sports urbains auto-organisés n ěst pas un choix hasardeux, puisque ce rapprochement est opéré dans la programmation des festivals de cultures urbaines, dans les catégories d intervention des politiques publiques de la culture (nationales ou locales), dans les discours des professionnel.le.s et des élu.e.s etc.

Dès lors qu on définit les cultures urbaines comme des pratiques masculines, les considérer comme un opérateur retourne le sens commun (qui en ferait les pratiques préférées des garçons) pour leur conférer une fonction de tri des garçons et des filles d une part (au détriment de celles-ci), du tri entre les garçons dominants et les autres d autre part. On peut donc poser 1 hypothèse que les cultures urbaines sont un « opérateur hiérarchique de genre » (Welzer-Lang, 2004 ; Ayral, 2009). Bien sûr cette fonction d opérateur est facilitée par une production médiatique abondante, mais la légitimité, lauatonomie et le statut qui sont conférés aux cultures urbaines dans les politiques publiques française (ville, jeunesse, culture) révèlent des «allants de soi», des « éléments tenus pour acquis » qui construisent la normalité des circonstances (Goffman, 1973), notamment celles qui initient la décision politique dans le cadre local: il va de soi que les problèmes viennent des garçons, en particulier des jeunes garçons immigrés. Il va de soi (dans un pays démocratique) que la gestion par la culture est préférable à la répression policière. Il va de soi que ces garçons immigrés représentent à la fois un danger et un potentiel qu il convient de valoriser. Mais aussi : il va de soi que les filles posent moins de problème, sauf lorsqu elles se mettent en danger en tant que femmes. Il va de soi que 1 `expression culturelle et sportive des filles n a que peu d importance. Il va de soi que le potentiel des filles doit être modéré en rapport avec leur possible fonction maternelleǔ .

La définition des «cultures urbaines » comme opérateur hiérarchique de genre et 1 inversion de sens que cette définition propose sont peu envisagées (à ma connaissance) par la recherche en sciences humaines et sociales. La variable sexe ne fait pas partie des variables utilisées couramment par les chercheur.e.s qui travaillent sur la sociologie urbaine ou 1 aménagement du territoire, on n énonce donc presque jamais que les «jeunes » que 1̌on

\footnotetext{
la construction des rapports de genre entre les :races $`$ noires et blanches, forgés par le colonialisme et la traite des esclaves.

12 «Le terme d’opérateur est généralement rapproché de celui d“actant, notion issue de la linguistique et transportée en sciences sociales ( ǔ ). Luactant y est caractérisé comme une réalité sociale, humaine ou non humaine, dotée d une capacité d`action. Par extension le mot en est venu à désigner toute entité définissable et distinguable qui participe à la dynamique et à ľorganisation d̆une action individuelle ou/et collective, qui est active dans un processus social, qui opère des actes. Les actants sont des :opérateurs de la vie sociale`[Lévy et Lussault, 2003], ainsi qualifiés en fonction de leur capacité à s insérer dans des relations sociales » (Ayral, 2009).
} 
nous présente comme les «nouvelles classes dangereuses » sont des garçonsǔ Cet impensé est $\mathrm{d}$ autant plus fort que de nouvelles préoccupations atteignent à présent le champ de la gestion urbaine et son « univers froid» qui le décrit comme un secteur important pour le contrôle social : il $\mathrm{n} \breve{y}$ a en effet qu un pas à franchir pour considérer que ces «nouvelles classes dangereuses », autrefois les classes populaires, sont issues de limmigration. La variable sexe est alors occultée par la variable ethnicité : peu importe que ce soient des garçons, il faut "s soccuper d eux» en particulier de ceux qui sont pauvres ou « issus de 1 immigration ». On peut expliquer ainsi le fait que la négation de la variable sexe consacre des cultures urbaines qui valorisent les garçons en général tout en stigmatisant les garçons des classes pauvres issus de 1 Łimmigration.

Cette hypothèse peut être illustrée par un exemple. Face au déficit dăanimateurs socioculturels de sexe masculin, notamment dans les quartiers où les équipements et les dispositifs encadrant les jeunes $n$ ăarivaient plus à contrôler les bandes de garçons devenus parfois seuls «maîtres à bord» de la maison commune (comme cela a été le cas à plusieurs reprises dans les centres danimation et les centres sociaux de la ville de Bordeaux), l embauche d animateurs «grands frères » a paru être la bonne solution. On peut penser, à 1 inverse, que la socialisation de garçons-issus-de-l immigration dans des cultures urbaines masculines, sous le contrôle d animateurs recrutés pour leur capital d ethnicité, n e est pas la meilleure solution pour diminuer la violence et 1̌ethnicisation des rapports de genreǔ A travers cet exemple (mais est-il besoin de rappeler à quel point il est étayé par une production médiatique foisonnante ?), on voit comment les cultures urbaines peuvent fonctionner, en bout de chaîne, comme opérateur hiérarchique non seulement de genre mais aussi de :racé.

La programmation par l 1 association Musique de Nuit du groupe de rap Sexion d Assaut à Cenon en Gironde en est un autre exemple. Alors que la tournée de ce groupe ouvertement homophobe avait été annulé dans 17 villes de France, le directeur de la salle et le maire socialiste de la ville ont décidé de maintenir le concert, contre 1 ̌avis des associations féministes et lesbiennes-gays-bi-trans de Bordeaux qui s expriment dans une pétition dont voici quelques extraits :

\section{Sexion d assaut}

«Malgré de nombreuses protestations, le concert du groupe Sexion d `Assaut est toujours prévu le samedi 23 octobre 2010 au Rocher de Palmer à Cenon (33). Ce groupe a tenu des propos violemment homophobes, appelant au meurtre $d$ homosexuels et incitant à la haine, 
dans des chansons et interviews («je crois quॅil est grand temps que les pédés périssent, coupe leur le pénis, laisse les morts, retrouvés sur le périphérique » (On ťa humilié, de lălbum La terre du milieu). Aujourd hui, le groupe prétend faire machine arrière mais il ne s agit que d excuses de circonstances destinées ni plus ni moins à sauver ses intérêts financiers. Jugeons plutôt les propos d un des leaders du groupe publiés en juin de cette année (magazine International Hip Hop, numéro 10) : «Pendant un temps, on a beaucoup attaqué les homosexuels parce qu ơn est homophobes à cent pour cent et qǔon l’assume. Mais on nous a fait beaucoup de réflexions et on šest dit qu il était mieux de ne plus trop en parler parce que ça pouvait nous porter préjudice [ǔ ] Imagine, il y a même des gays qui viennent nous voir! On ne peut donc pas dire ouvertement que pour nous, le fait d’être homosexuel est une déviance qui ňest pas tolérable ».(ǔ ). Les associations signataires s indignent que le maire de Cenon et le directeur du Rocher de Palmer maintiennent toujours le concert du 23 octobre. Les propos de Patrick Duval, responsable de la salle, relatés par le journal SudOuest le 9 octobre 2010, nous choquent : «Čest une déclaration dans une publication confidentielle [ǔ ] mais il ne faut pas être dupe : cela correspond à ce qui se dit beaucoup dans les quartiers. ».

Dans un autre communiqué au journal Sud-Ouest Patrick Duval plaide a posteriori l utilité du débat ouvert par la venue de Sexion d`Assaut pour un public de «jeunes des quartiers » qui seraient eux-mêmes souvent homophobes, et traite les responsables des associations qui demandent l`annulation du concert de « voyous » pour ne pas avoir demandé un rendez-vous avec lui pour en discuter. D autres programmateurs des musiques actuelles appartenant au Réseau Aquitain des Musiques Amplifiées défendent la venue de Sexion d`Assaut au nom de la liberté d expression et de l⿳antiracisme (le groupe est composé de musiciens issus de l־immigration et/ou à la peau noire ou foncée), fustigeant le « politiquement correct» des associations féministes et lgbt. Il est vrai que les mêmes avaient fêté un mois plus tôt avec enthousiasme le retour sur la scène bordelaise de Bertrand Cantat, le chanteur du groupe rock Noir Désir, condamné à huit ans de prison pour le meurtre de sa compagne.

\section{Conclusion}

Les cultures urbaines sont devenues des opérateurs de la vie sociale par le sens que 
leur ont progressivement donné les chaînes d acteurs qu ělles mobilisent dans les salles de répétition, sur les terrains de sport, sur les murs de la ville, dans les espaces publics, dans les manifestations culturelles, dans les médias etc. Enoncer le fait que les cultures urbaines sont des cultures masculines permet de comprendre comment émergent de nouveaux modèles de virilité qui font consensus en un temps et sur des lieux donnés. On peut poser 1hypothèse que lassociation entre cultures urbaines et territoires imaginaires de banlieue «exotise» ces cultures viriles en leur offrant de nouveaux espaces où peuvent se redéployer le sexisme et 1 homophobie, dont lexpression est ailleurs contenue par la régulation démocratique des rapports hommes-femmes et le respect des libertés individuelles. Les cultures urbaines sont donc probablement 1 objet d un compromis, tolérant au-delà de la frontière du ghetto ce qui est interdit en deçà. Les mythes légendaires des cultures de banlieue (la marge tient la pageǔ éloge du métissage et de 1 interculturalitéǔ régulation sociale par la cultureǔ ) apparaissent sous 1 ăngle de lévolution des rapports sociaux de sexe parfaitement réactionnaires. On peut craindre en effet que leur incrustation dans les politiques publiques de la prévention, de la jeunesse et de la culture favorise dune part la constitution de lieux de reproduction des attitudes et des valeurs viriles (et leur diffusion à l`ensemble de la société), d autre part la mise à lécart des territoires symboliques auxquels ces lieux se réfèrent, et pour les mêmes raisons. Dans ces conditions, ce qui est généralement présenté comme une politique publique tolérante et progressiste pourrait aussi bien être analysé comme participant au contraire dune « logique de 1 exclusion» (Elias et Scotson, 19997 [1965]), la mise en place d un contrôle culturel symbolique des quartiers agissant de façon impensée dans le sens du maintien des rapports de domination de genre et de :race à 1 `intérieur d espaces prescrits.

\section{Références bibliographiques}

AYRAL, S. 2009. La fabrique des garçons. Sanction et genre au collège. Thèse de doctorat s.d E. Debarbieux, Université Segalen, Bordeaux.

BECKER, H. 1985 [1963]. Outsiders, Etude de sociologie de la déviance, Paris, Métaillé. BOURDIEU, P. 1998. La domination masculine, Paris Ed. de Minuit.

COUTRAS, J. 2003. Les peurs urbaines et ľautre sexe, Paris, L Harmattan.

DORLIN, E. 2006. La matrice de la race, généalogie sexuelle et coloniale de la nation française, Paris, La Découverte.

FELONNEAU, M.-L. 2001. «Les tags sur les murs de nos villes », in Agora Débat jeunesse $n^{\circ} 24$, Paris, L `Harmattan, p.63-73. 
FELOUZIS, G.; LIOT, F. ; PERROTON, J. 2005. L Lapartheid scolaire. Enquête sur la ségrégation ethnique dans les collèges, Paris, Seuil.

GARFINKEL, H. 2007 [1967]. Recherches en ethnométhodologie, Paris, PUF.

GODELIER, M. 1988. « Trahir le secret des hommes », Le Genre Humain n 17.

ELIAS, N. ; SCOTSON, J. 1997 [1965]. Logiques de l'exclusion, Paris, Arthème Fayard.

GOFFMAN, E. 2002 [1977]. L a arrangement des sexes, Paris, La Dispute.

KEBABZAH, H. 2004. « La ségrégation sexuée dans les quartiers populaires », in Les filles et les garçons sont-ils éduqués ensemble?, Ville, école, intégration, diversité ${ }^{\circ} 138$, CNDPCRDP, p.129-136.

LAPEYRONNIE, D. 2008. Ghetto urbain, Ségrégation, violence, pauvreté en France aujourd hui, Paris, Robert Laffont.

LATOUR, B. 1989. La science en action, Paris, La Découverte.

LEVY, J. ; LUSSAULT, M. 2003. Dictionnaire de la géographie, Paris, La Découverte.

LUSSAULT M., L־Homme spatial. La construction sociale de ľespace humain, Paris, Seuil.

MENDJELI, R. 2003. «Eléments pour une analyse du discours sur le logement et 1 intégration des populations immigrées », in Boumaza et al. Relations interethniques dans lhabitat et dans la ville. Agir contre les discriminations promouvoir les cultures résidentielles, Paris, Ed, 1 Harmattan, 2003, p. 101-118.

MENDJELI, R. ; RAIBAUD, Y. 2008. «Politique de la ville et construction de limage ethnique », Copyright Volume ${ }^{\circ} 10$, Angers, éd. Seteun, p. 81-93.

RAIBAUD, Y. 2005a. «Des lieux construits par le genre», Géographie et Culture, n54, p.53-70.

RAIBAUD, Y. 2005b. Territoires musicaux en région, Pessac, éd. MSHA.

RAIBAUD, Y. 2007a. Genre et loisirs des jeunes, in Des femmes et des hommes : un enjeu pour le social? Empan $\mathrm{n}^{\circ} 65$, p. 67-73

RAIBAUD, Y. 2007b. Le genre et le sexe comme objet géographique, Cahier ADES, HAL Archives ouvertes du CNRS.

WELZER-LANG, D. 2004. Les hommes aussi changent, Paris, Payot. 